\title{
Low Temperature Scanning Electron Microscopy of Irregular Snow Crystals
}

\author{
William P. Wergin, ${ }^{*}$ Albert Rango, ${ }^{* *}$ James Foster, ${ }^{* * *}$ Eric F. Erbe, ${ }^{*}$ and Christopher Pooley****
}

*Soybean Genomics and Improvement Laboratory, Agricultural Research Service (ARS), U.S. Department of Agriculture (USDA), Beltsville, MD 20705 USA

**Jornada Experimental Range, ARS, USDA, New Mexico State University, 401 E. College Ave., Las Cruces, NM 88003 USA

***Laboratory for Hydrological Sciences, NASA Goddard Space Flight Center, Greenbelt, MD 20771 USA

****Hydrology and Remote Sensing Laboratory and Soybean Genetics and Improvement Laboratory, ARS, USDA, Beltsville, MD 20705 USA

Snow crystals occur in eight basic types: columns, needles, plates, dendrites, irregular crystals, graupel, hail and ice pellets.[1] Most of these types have been described and photographed.[2] However, our understanding of the "irregular crystals" remains vague because the limited resolution and depth-of-field associated with the light microscope have prevented investigators from fully characterizing and clearly illustrating the features of these crystals. Our study used a field-emission scanning electron microscope (FESEM), equipped with a cold stage, to document the structural features, physical associations and atmospheric metamorphosis of irregular snow crystals.

Snow samples, consisting of freshly fallen snowflakes, were collected from six different locations where air temperatures ranged from $-5^{0} \mathrm{C}$ to $0^{0} \mathrm{C}$. The collection procedure consisted of spreading a cryo-adhesive on a pre-cooled flat copper plate and allowing newly fallen snowflakes to settle on the surface. Next, the plate was rapidly frozen in $\mathrm{LN}_{2}$. The frozen plates were placed in a dry shipping dewar and conveyed to the laboratory where they were coated for observation in a Hitachi S-4100 FESEM equipped with an Oxford CT 1500 HF Cryotrans system as previously describe.[3]

Crystals, believed to correspond to irregular crystals, appeared as short, irregular hexagons, measuring 60 to $90 \mu \mathrm{m}$ across, when viewed from the a-axis (Fig. 1). Their length (c-axis) rarely exceeded the diameter. The irregular crystals were occasionally found as secondary particles on other larger forms of snow crystals (Fig. 2). However, they most frequently occurred as aggregates consisting of more than 100 irregular crystals (Fig. 3). In the aggregates, the irregular crystals had their c-axes oriented parallel to one another and collectively, tended to form columnar structures. Occasionally the aggregates exhibited rounded facets along one side, suggesting atmospheric metamorphoses and unidirectional fall. In extreme cases of metamorphoses, the aggregates would be difficult to distinguish from graupel. Frost, consisting of irregular crystals, was also encountered, suggesting that atmospheric conditions that favor this form of growth also occur terrestrially (Fig. 4).

Results obtained with low temperature FESEM suggested that crystals, which were previously designated as "irregular crystals", have distinctive features that can be used to characterize and distinguish them from other types of snow crystals.

[1] S. Colbeck et al., The International Classification for Seasonal Snow on the Ground, Univ. of Colorado, Boulder, 1990.

[2] U. Nakaya. Snow Crystals: Natural and Artificial, Harvard Univ. Press, Cambridge, MA, 1954.

[3] W.P. Wergin et al., Scanning. 17 (1995) 41. 


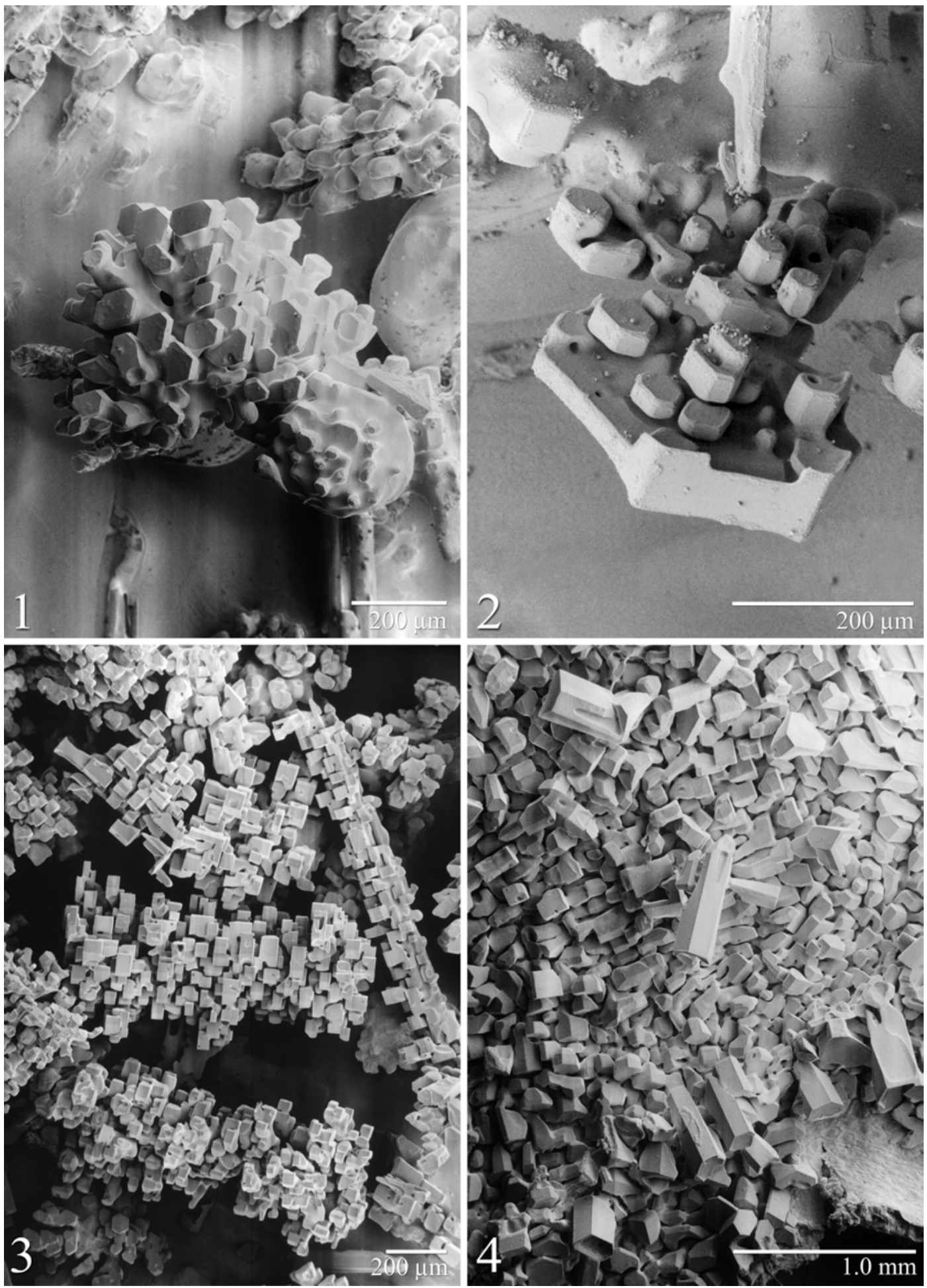

Figure 1. A-axis view of irregular crystals, which appear as asymmetric hexagons.

Figure 2. Irregular crystals, which occur as secondary particles on the surface of a dendritic arm.

Figure 3. Several "snowflakes", each consisting of aggregates of numerous irregular crystals.

Figure 4. Irregular crystals that had formed on the surface of a leaf. 